\title{
PD-L1 expression in EBV-negative diffuse large B-cell lymphoma: clinicopathologic features and prognostic implications
}

\author{
Wei Xing ${ }^{1}$, Karen Dresser ${ }^{1}$, Rui Zhang ${ }^{2}$, Andrew M. Evens ${ }^{3}$, Hongbo Yu ${ }^{1,4}$, Bruce A. \\ Woda $^{1}$, Benjamin J. Chen ${ }^{1}$ \\ ${ }^{1}$ Department of Pathology, UMass Memorial Medical Center and University of Massachusetts Medical School, Worcester, MA, USA \\ ${ }^{2}$ Department of Biostatistics, Boston University School of Public Health, Boston, MA, USA \\ ${ }^{3}$ Division of Hematology/Oncology, Tufts Medical Center, Boston, MA, USA \\ ${ }^{4}$ Present Address: Department of Pathology and Laboratory Medicine, VA Boston Healthcare System, West Roxbury, MA, USA \\ Correspondence to: Benjamin J. Chen, email: benjamin.chen@umassmemorial.org \\ Keywords: programmed cell death ligand 1, diffuse large B-cell lymphoma, immune checkpoint, immunotherapy \\ Received: December 28, $2015 \quad$ Accepted: July 18, $2016 \quad$ Published: August 04, 2016
}

\section{ABSTRACT}

Programmed cell death ligand 1 (PD-L1) is a cell surface glycoprotein that regulates the cellular immune response and serves as a targetable immune checkpoint molecule. PD-L1 is expressed on tumor cells and the immune microenvironment of several human malignancies, including a subset of aggressive lymphomas. We sought to investigate further the clinical and pathologic features of EBV-negative diffuse large B-cell lymphoma (DLBCL) cases that express PD-L1. Immunohistochemical staining using an anti-PD-L1 monoclonal antibody was performed on DLBCL cases from 86 patients. These patients received standard chemotherapy treatment and were followed for up to 175 months. Overall, 14 cases (16\%) were considered positive for PD-L1 in tumor cells. In comparison with PD-L1 negative cases, PD-L1 positive cases had a higher rate of non-GCB type (71\% vs. 30\%, $P=0.0060)$, and higher Ann Arbor stage (II-IV) $(100 \%$ vs. $73 \%, P=0.0327)$. No significant differences were seen in the immunohistochemical expression of BCL2, MYC, or Ki67. Patients with tumors expressing PD-L1 demonstrated inferior overall survival (OS) upon long term follow up $(P=0.0447)$. Both age/sex-adjusted and multivariate analyses identified PD-L1 as an independent predictor for $O S(P=0.0101$ and $P=0.0424)$. There was no significant difference, however, in terms of remission rates after first treatment, relapse rates, and progression free survival between the groups. Identification of DLBCL cases that express PD-L1 may serve to select a subset of patients that could further benefit from targeted immunotherapy.

\section{INTRODUCTION}

Immune checkpoint blockade strategies have revolutionized the approach to cancer therapy and have provided oncologists and patients with novel therapeutic options for a range of malignancies [1]. Immunotherapeutic targeting of hematologic malignancies, most notably classical Hodgkin lymphoma, have recently centered on disrupting the programmed death-1 (PD-1) immunomodulatory pathway $[2,3]$. Binding of PD-1 by its cognate receptors PD-L1 and PD-L2 inhibits proliferation of activated T cells in peripheral tissues leading to "T-cell exhaustion," a functional phenotype that can be reversed by PD-1 blockade [4]. Immunohistochemical detection of PD-L1 has been well-documented in a range of human malignancies, including aggressive EBV-positive B-cell lymphomas [5], classical Hodgkin lymphoma [6, 7], melanoma, renal cell carcinoma, and non-small cell lung carcinoma [8]. Interestingly, PD-L1 expression has been observed on tumor cells as well as on non-malignant infiltrating histiocytes, suggesting tumors may elicit an overall immunosuppressive microenvironment as a means of tumorigenesis [5].

These findings have provided a rationale for disrupting the PD-1 axis using antibodies against these antigens with the objective of restoring the anti- 
tumor activity of suppressed T cells. Clinical trials with humanized monoclonal antibodies against PD-L1 and PD-1 have yielded robust, durable responses in patients with advanced malignancies including metastatic melanoma, tumors with mismatch-repair deficiency, and relapsed or refractory classical Hodgkin lymphoma [912]. Responses have also been reported in smaller series of hematologic malignancies, including acute myeloid leukemia, follicular lymphoma, and a number of cases of diffuse large B-cell lymphoma (DLBCL) [13-15]. Notably, in a subset of cases examined, clinical responsiveness to PD-1 blockade correlated with tumor-specific expression of PD-L1 as detected by immunohistochemistry (IHC) [8, 12].

DLBCL, not otherwise specified (NOS), is the most common type of non-Hodgkin lymphoma among adults and is both clinically and pathologically heterogeneous. Several pathologic features are known to predict a worse clinical outcome for DLBCL patients, including non-germinal center B-cell (non-GCB) phenotype [16], increased MYC protein expression [17], and gene rearrangements involving $B C L 2, B C L 6$, and/or $M Y C$ [1820]. Identifying additional features that could predict potential response to immunotherapy is important to both understanding the immunomodulatory mechanisms of hematologic malignancies and to developing novel chemotherapeutic regimens.

In the present study, we sought to investigate further the clinical and pathologic features of EBV-negative DLBCL cases with PD-L1 expression. Using a well- annotated cohort of patients with treatment information and long term clinical follow-up, we examined the immunohistochemical expression of PD-L1 in tumor cells and the microenvironment, and correlated these data with additional histologic parameters and clinical outcome data.

\section{RESULTS}

\section{Pathologic characteristics}

PD-L1-positive tumor cells, along with PDL1-positive non-malignant cells, were recorded as a percentage of the total cellularity within tumor sections (Figure 1). A threshold of $>30 \%$ PD-L1-positive tumor cells captured the majority of cases that exhibited positive PD-L1 staining $(14 / 27,52 \%)$, similar to the findings of a recent study [21]. Of the 86 DLBCL cases, 14 cases (16\%) were considered PD-L1 positive cases based on this threshold. Of the 72 PD-L1 tumor negative cases, 23 cases $(27 \%)$ showed at least $5 \%$ PD-L1 positive staining in the non-malignant cells (mPD-L1 positive, Figure 1). Representative photographs of DLBCL cases stained with PD-L1 IHC with corresponding scores are shown in Figure 2. Eighty-three cases had evaluable material to be further subcategorized into GCB and non-GCB types according to the cell-of-origin (COO) Hans algorithm [16]. Out of 14 PD-L1 positive cases, 4 cases (29\%) were GCB type and 10 cases $(71 \%)$ were non-GCB type. Out of 69 PDL1 negative cases, 48 cases $(70 \%)$ were GCB type and 21

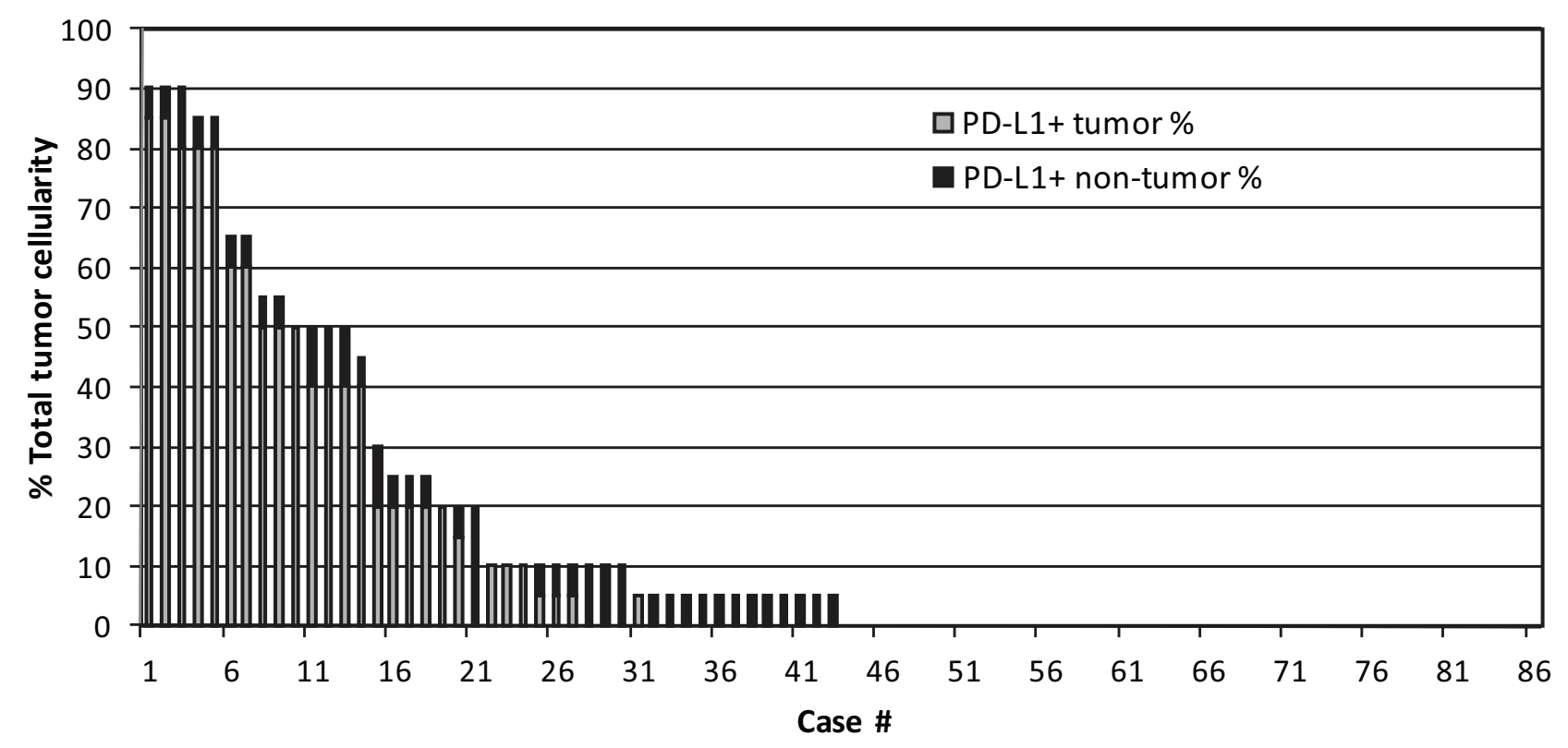

Figure 1: PD-L1 scores in lymphoma cells and microenvironment of 86 DLBCL cases. Graphical representation of the percentage of total cells within the tissue section staining positive with anti-PD-L1, and showing the contributions of malignant cells (white bars) and non-malignant cells (black bars) in each case of DLBCL. 

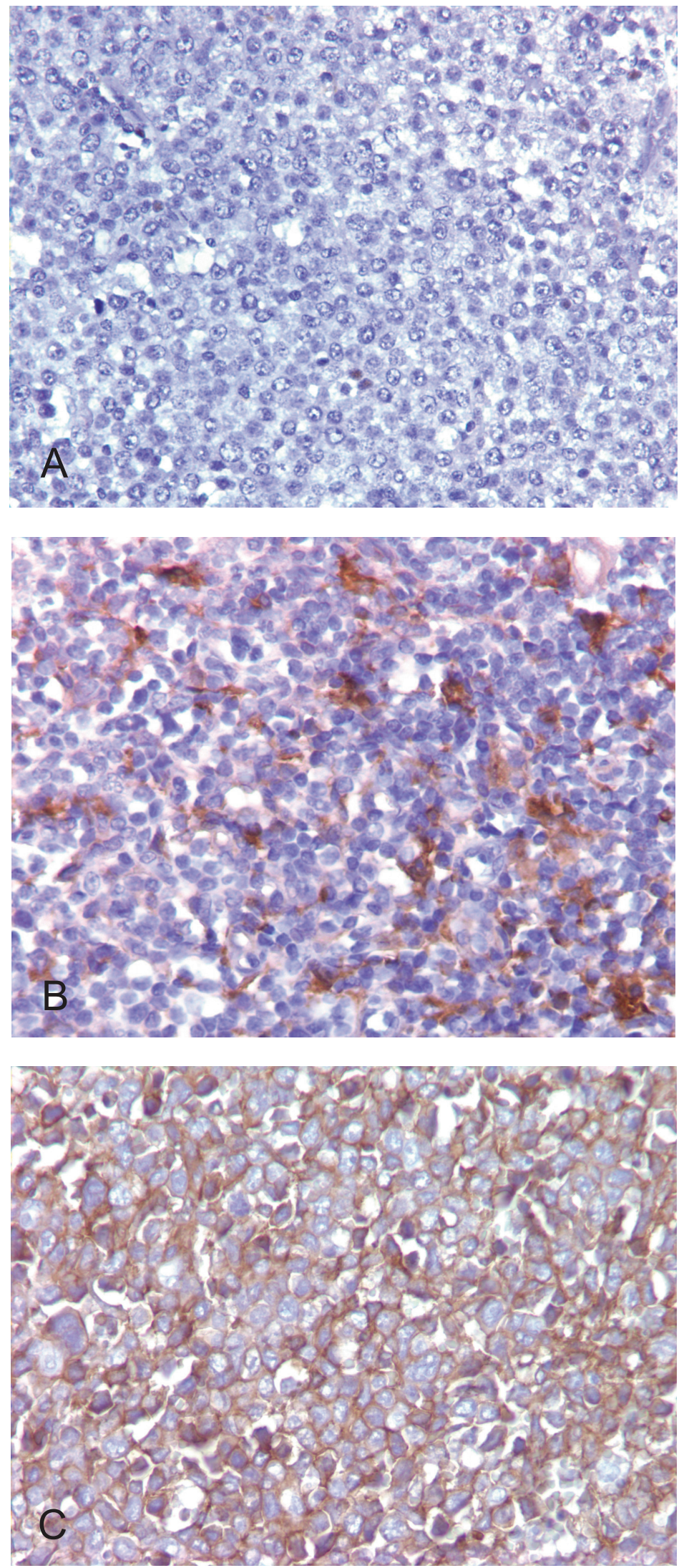

Figure 2: PD-L1 expression detected by immunohistochemical staining in DLBCL. A. Negative staining. B. Tumor cells negative for PD-L1 but histiocytes in the microenvironment staining positive, about $10 \%$ of the total cellularity. C. Tumor cells showing strong membrane positivity for PD-L1. Original magnification: 400x. 
Table 1: Pathologic features of DLBCL cases with PD-L1 expression

\begin{tabular}{|c|c|c|c|c|c|c|c|}
\hline \multirow{2}{*}{ Pathologic Features } & \multirow{2}{*}{ PD-L1 positive } & \multicolumn{2}{|c|}{ PD-L1 negative } & \multirow{2}{*}{ Total } & \multirow{2}{*}{$\mathbf{P}^{\Phi}$} & \multirow{2}{*}{$\mathbf{P}^{\#}$} & \multirow{2}{*}{$\mathbf{P}^{\dagger}$} \\
\hline & & mPD-L1-positive & mPD-L1-negative & & & & \\
\hline \multicolumn{8}{|l|}{ Phenotype } \\
\hline GCB & $4(29 \%)$ & $13(57 \%)$ & $35(76 \%)$ & 52 & $0.0060^{*}$ & 0.1067 & $0.0027^{*}$ \\
\hline Non-GCB & $10(71 \%)$ & $10(43 \%)$ & $11(24 \%)$ & 31 & & & \\
\hline \multicolumn{8}{|l|}{ BCL2 } \\
\hline Positive (>30\%) & $11(79 \%)$ & $15(71 \%)$ & $24(51 \%)$ & 50 & 0.2283 & 0.1841 & 0.1220 \\
\hline Negative & $3(21 \%)$ & $6(29 \%)$ & $23(49 \%)$ & 32 & & & \\
\hline \multicolumn{8}{|l|}{ BCL6 } \\
\hline Positive (>30\%) & $5(36 \%)$ & $16(76 \%)$ & $32(70 \%)$ & 53 & $0.0146^{*}$ & 0.7714 & $0.0306^{*}$ \\
\hline Negative & $9(64 \%)$ & $5(24 \%)$ & $14(30 \%)$ & 28 & & & \\
\hline \multicolumn{8}{|l|}{ CD10 } \\
\hline Positive & $1(7 \%)$ & $7(32 \%)$ & $23(49 \%)$ & 31 & $0.0134 *$ & 0.2041 & $0.0050^{*}$ \\
\hline Negative & $13(93 \%)$ & $15(68 \%)$ & $24(51 \%)$ & 52 & & & \\
\hline \multicolumn{8}{|l|}{ MUM1 } \\
\hline Positive (>30\%) & $13(93 \%)$ & $12(55 \%)$ & $21(48 \%)$ & 46 & $0.0029 *$ & 0.7944 & $0.0040^{*}$ \\
\hline Negative & $1(7 \%)$ & $10(45 \%)$ & $23(52 \%)$ & 34 & & & \\
\hline \multicolumn{8}{|l|}{ MYC } \\
\hline$<40 \%$ & $7(50 \%)$ & $11(48 \%)$ & $34(74 \%)$ & 52 & 0.3659 & 0.0590 & 0.1108 \\
\hline$\geq 40 \%$ & $7(50 \%)$ & $12(52 \%)$ & $12(26 \%)$ & 31 & & & \\
\hline \multicolumn{8}{|l|}{ Ki67 } \\
\hline$<80 \%$ & $10(71 \%)$ & $14(61 \%)$ & $34(71 \%)$ & 58 & 0.7789 & 0.4275 & 1.0000 \\
\hline$\geq 80 \%$ & $4(29 \%)$ & $9(39 \%)$ & $14(29 \%)$ & 27 & & & \\
\hline
\end{tabular}

Fisher's exact test. *: P value is significant $(<0.05) ; \mathrm{P}^{\Phi}$ : PD-L1-positive versus PD-L1-negative; $\mathrm{P}^{\#}$ : mPD-L1-positive versus mPD-L1-negative; $\mathrm{P}^{\dagger}$ : PD-L1-positive versus mPD-L1-negative.

cases $(30 \%)$ were non-GCB type. PD-L1 positive cases had a higher rate of non-GCB phenotype compared to PDL1 negative cases ( $71 \%$ vs. $30 \%, \mathrm{P}=0.0060$, Table 1$)$.

The overall positivity rates of CD10, BCL2, BCL6, and MUM1 were $36 \%, 58 \%, 62 \%$, and $53 \%$, respectively. MYC positive tumor cells by IHC ranged from $0 \%$ to $80 \%$ (mean \pm SD, $28.3 \pm 25.3 \%$ ). The Ki67 labeling index ranged from $10 \%$ to $95 \%$ (mean $\pm \mathrm{SD}, 56.2 \pm 26.2 \%$ ). CD10, BCL6, and MUM1 showed significant differences between the PD-L1 positive and negative groups reflecting the non-GCB vs. GCB phenotype differences (Table 1). When comparing the immunohistochemical expression of BCL2, MYC, or Ki67 between the PD-L1 positive and PD-L1 negative groups, no significant differences were seen (Table 1). Separate analysis of MPD-L1 positive vs mPD-L1 negative cases showed no significant differences in these IHC studies.

\section{Clinical characteristics}

Clinical features including age, gender, presence of B symptoms, pre-treatment LDH, IPI score, and bone marrow involvement showed no statistical difference between PD-L1 positive and negative groups (Table 2). The total extralymphatic organ (organs other than bone marrow, lymph node and spleen) involvement rate was $59 \%$ (46/78, Table 2). Both the PD-L1 tumor positive group (10/12, 83\%) and mPD-L1 positive group (17/20, $85 \%$ ) showed greater extralymphatic organ involvement compared to the mPD-L1 negative group (19/46, 41\%), which was statistically significant $(\mathrm{P}=0.0206$ and $\mathrm{P}=0.0012$ respectively, Table 2). The rates of high Ann Arbor stage (II-IV) at diagnosis of mPD-L1 negative, mPD-L1 positive and PD-L1 positive cases were $67 \%$ (30/45), 86\% (18/21) and 100\% (13/13), respectively. PD- 
Table 2: Clinical features of DLBCL cases with PD-L1 expression

\begin{tabular}{|c|c|c|c|c|c|c|c|}
\hline \multirow{2}{*}{ Clinical Features } & \multirow{2}{*}{ PD-L1 positive } & \multicolumn{2}{|c|}{ PD-L1 negative } & \multirow{2}{*}{ Total } & \multirow{2}{*}{$\mathbf{P}^{\Phi}$} & \multirow{2}{*}{$\mathbf{P}^{\#}$} & \multirow{2}{*}{$\mathbf{P}^{\dagger}$} \\
\hline & & mPD-L1-positive & mPD-L1-negative & & & & \\
\hline \multicolumn{8}{|l|}{ Age (years) } \\
\hline$\leq 60$ & $5(36 \%)$ & $5(22 \%)$ & $14(29 \%)$ & 24 & 0.5216 & 0.7749 & 0.7428 \\
\hline$>60$ & $9(64 \%)$ & $18(78 \%)$ & $35(71 \%)$ & 62 & & & \\
\hline
\end{tabular}

Gender

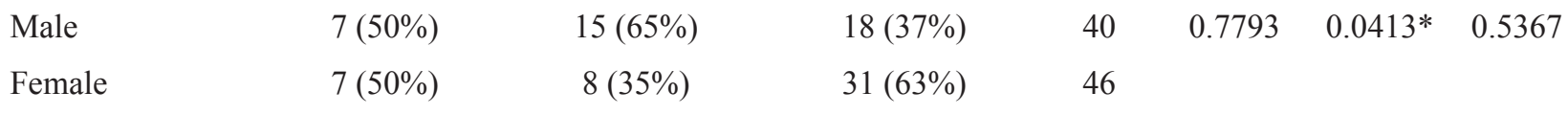

B symptoms

Presence

Absence

$2(25 \%)$

$6(75 \%)$

$3(18 \%)$

$14(82 \%)$

$12(33 \%)$

$24(67 \%)$

17

1.0000

0.3332

1.0000

Pre-treatment LDH

Normal

$2(20 \%)$

$11(58 \%)$

$8(80 \%)$

$8(42 \%)$

$1(10 \%)$

$7(41 \%)$

$9(90 \%)$

$10(59 \%)$

$13(30 \%)$

$31(70 \%)$

$3(14 \%)$

$18(86 \%)$

$15(33 \%)$

$30(67 \%)$

$3(19 \%)$

$13(81 \%)$

$2(6 \%)$

$30(94 \%)$

8

0.1309

$0.3164 \quad 0.0782$

Absent

$7(70 \%)$

Extralymphatic involvement

Present

$\begin{array}{ll}10(83 \%) & 17(85 \%) \\ 2(17 \%) & 3(15 \%)\end{array}$

Absent

$2(17 \%)$

$3(15 \%)$

$19(41 \%)$

$27(59 \%)$

46

32

Complete and partial remission after first treatment

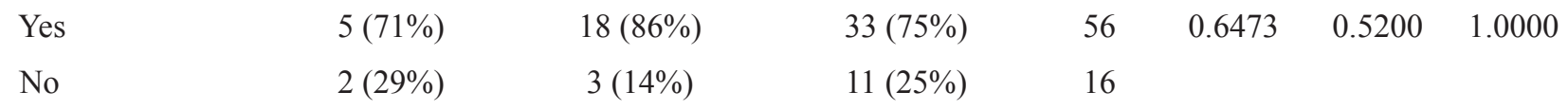

Relapse after initial treatment

\begin{tabular}{|c|c|c|c|c|c|c|}
\hline Yes & $9(82 \%)$ & $10(56 \%)$ & $31(69 \%)$ & 50 & 0.4861 & 0.3850 \\
\hline No & $2(18 \%)$ & $8(44 \%)$ & $14(31 \%)$ & 2 & & \\
\hline
\end{tabular}

Outcome

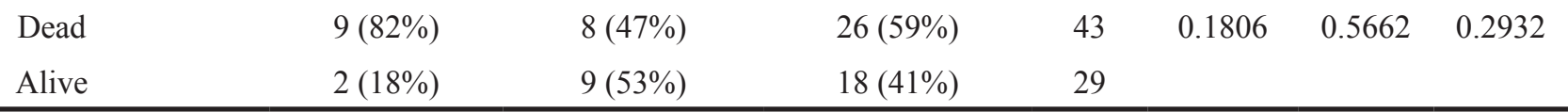

Fisher's exact test. *: P value is significant $(<0.05)$; $\mathrm{P}^{\Phi}$ : PD-L1-positive versus PD-L1-negative; $\mathrm{P}^{\#}$ : mPD-L1-positive versus mPD-L1-negative; $\mathrm{P}^{\dagger}$ : PD-L1-positive versus mPD-L1-negative. 
L1 positive cases had significantly higher initial staging than PD-L1 negative cases $(\mathrm{P}=0.0327)$.

The majority of patients $(73 / 86,85 \%)$ received rituximab, cyclophosphamide, doxorubicin, vincristine and prednisone (R-CHOP) as standard chemotherapy. Other treatment regimens included dose-adjusted etoposide, vincristine, doxorubicin, cyclophosphamide and prednisone $(\mathrm{EPOCH})$; hyper-fractionated cyclophosphamide, vincristine, doxorubicin and dexamethasone (hyperCVAD); chlorambucil plus prednisone; rituximab alone; methotrexate alone; and radiation alone. None of the patients received hematopoietic stem cell transplant. All patients were followed by clinical and radiological assessments over a period ranging from 2 days to 175 months (median follow-up 21 months).

Of 72 patients who received R-CHOP treatment and had available followup information, the overall response rate (complete and partial remission) was 78\% (56/72). No significant differences were found among the three groups (Table 2).

After initial treatment, the median progression-free survival (PFS) time was 18.5 months (range, 2 days to 173 months) and the median overall survival (OS) time was 21 months (range, 2 days to 174 months). For survival analyses, the 86 cases were divided into 2 groups: PD-L1 positive and PD-L1 negative groups (including $\mathrm{MPD}-\mathrm{L} 1$ positive and mPD-L1 negative cases). Kaplan-Meier curves of PFS and OS showed apparent decreased survival of the PD-L1 positive group (Figure 3), although only the difference in OS was statistically significant by Log Rank test $\left(\mathrm{P}_{\mathrm{PFS}}=0.0773 ; \mathrm{P}_{\mathrm{OS}}=0.0447\right)$. Within the PD-L1 negative group, there was no significant difference in OS between the mPD-L1+ and mPD-L1- subgroups (data not shown).
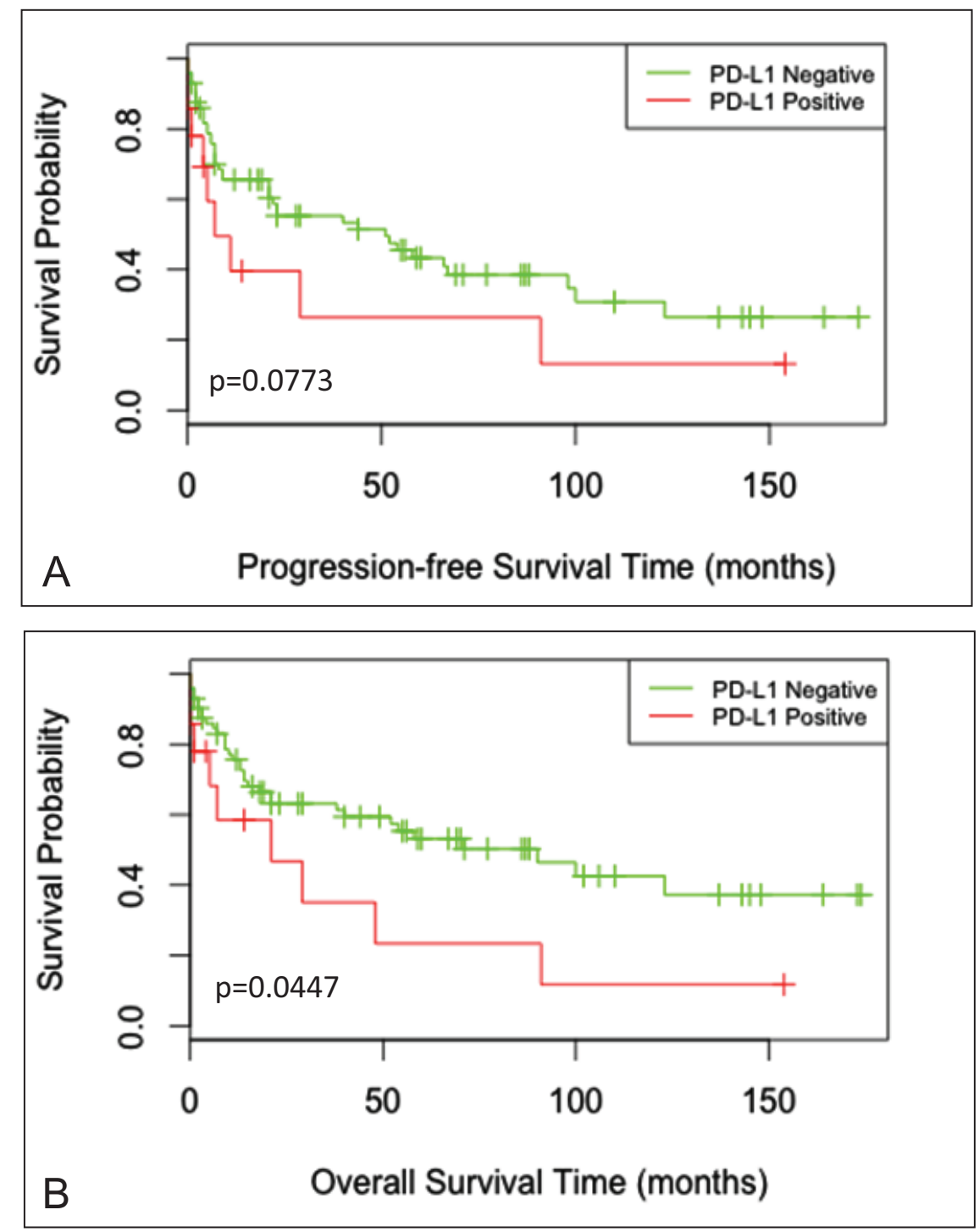

Figure 3: Survival curves for progression-free survival and overall survival. Kaplan-Meier curves for A. progression-free survival ( $\mathrm{PFS}$ ) time ( $\mathrm{P}=0.0773$ ) and $\mathbf{B}$. overall survival $(\mathrm{OS})$ time ( $\mathrm{P}=0.0447)$ among PD-L1 positive and PD-L1 negative cases. 
Table 3: Prognostic factors affecting the OS of patients with EBV- DLBCL

\begin{tabular}{|c|c|c|c|c|}
\hline \multirow{2}{*}{ Characteristic } & \multicolumn{2}{|c|}{ Age- and sex-adjusted analysis } & \multicolumn{2}{|c|}{ Multivariate analysis ${ }^{\#}$} \\
\hline & HR $(95 \%$ CI $)$ & $P$ & HR $(95 \%$ CI $)$ & $\boldsymbol{P}$ \\
\hline PD-L1+ vs. PD-L1- & $2.740(1.272-5.904)$ & $0.0101 *$ & $2.418(1.031-5.670)$ & $0.0424 *$ \\
\hline Non-GCB vs. GCB & $1.824(0.962-3.460)$ & 0.0657 & $1.321(0.640-2.730)$ & 0.4517 \\
\hline Stage II-IV vs. Stage I & $4.318(1.508-12.362)$ & $0.0064 *$ & $3.125(0.880-11.101)$ & 0.0781 \\
\hline $\begin{array}{l}\text { IPI intermediate-high } \\
\text { vs IPI low }\end{array}$ & $3.949(1.353-11.523)$ & $0.0120 *$ & $2.961(0.823-10.658)$ & 0.0967 \\
\hline
\end{tabular}

*: P value is significant $(<0.05)$.

\#: The variables included in multivariate analysis for OS were age, sex, expression of PD-L1, cell of origin, stage, and IPI.

Age and sex-adjusted Cox regression analysis of prognostic factors for OS revealed PD-L1 expression $(\mathrm{P}=0.0101)$, high stage (II-IV) $\quad(\mathrm{P}=0.0064)$, and intermediate-high IPI $(2-5)(\mathrm{P}=0.0120)$ were statistically significant predictors of OS (Table 3 ). Non-GCB type was also an unfavorable factor with borderline statistical significance $(\mathrm{P}=0.0657)$. In a multivariate model examining these factors, PD-L1 expression remained a statistically significant factor for $\operatorname{OS}(\mathrm{P}=0.0424)$ (Table 3$)$.

\section{DISCUSSION}

Immune checkpoint blockade therapy is a promising chemotherapeutic strategy that will likely lead to novel multimodal and combinatorial approaches to treating hematologic malignancies [1]. In the case of B-cell nonHodgkin lymphoma, limited but encouraging results have been observed in early clinical trials [13-15]. In many of the recent clinical trials of anti-PD1/PDL1 immunotherapy, PD-L1 expression in tumor cells was not used as a prerequisite for patient enrollment. Retrospective analyses, however, have shown that PD-L1 positivity in tumor cells, as detected by immunohistochemistry, may predict improved response to anti-PD1/PD-L1 therapy in melanoma, non-small cell lung carcinoma, renal cell carcinoma, and urothelial carcinoma [8, 12, 22]. Thus, profiling tumors for PD-L1 expression may aid in the rational selection of patients for whom immunotherapy may be expected to provide a more robust response.

In this study, we correlated PD-L1 expression in EBV-negative DLBCL cases with clinical and pathologic features. In our cohort, $16 \%$ of cases were positive for PD-L1 in tumor cells and an additional $27 \%$ had positive microenvironment staining. Several recent studies have examined the incidence of PD-L1 positivity in DLBCL, NOS cases and variants of DLBCL, including T-cell/ histiocyte-rich large B-cell lymphoma and EBV-positive DLBCL of the elderly [5, 21, 23-25]. The proportion of DLBCL, NOS cases in our study that was positive for PDL1 was comparable to that seen in these previous studies examining similar cohorts of DLBCL cases. Furthermore, we confirm previous observations that PD-L1 positive DLBCL cases were more commonly of the non-germinal center B-cell type, which typically portends a poorer overall prognosis. In addition, PD-L1 expression was not significantly associated with other prognostic features such as BCL2 or Ki67 proliferation index.

In addition to non-GCB phenotype, we found that patients with PD-L1 positive DLBCL had higher-stage tumors than those with PD-L1 negative DLBCL. We also found a trend towards poorer outcomes in terms of PFS and OS for patients with PD-L1 positive tumors. Decreased OS was found to be statistically significant in our cohort. Two analyses of clinical outcome in PD-L1 positive DLBCL have recently been reported [21, 25]. Kiyasu, et al [21], examined a cohort of 1253 DLBCL cases and found that, in a smaller subset of cases with available clinical data (273 cases), PD-L1 positivity in tumor cells correlated with poorer overall survival. This cohort included cases of DLBCL, NOS, as well as variants such as EBV+ DLBCL. In this study, a threshold of $>30 \%$ of tumor cells positive for PD-L1 was identified based upon the observation that the majority of cases were captured by this analysis. In the current study, we find that a similar threshold appears to identify a cohort of DLBCL cases that also have poorer overall survival providing an important external validation of the prior findings and supporting the use of this threshold. Another recent study by Kwon, et al [25], examined 126 DLBCL cases. This cohort included cases of DLBCL, NOS, as well as EBV+ DLBCL cases. In this study, a threshold of 10\% PD-L1 positivity was used, yielding about $30 \%$ of DLBCL cases with at least moderate staining. No significant correlation with patient outcome was seen in this analysis.

Among the studies examining PD-L1 expression in DLBCL, it should be noted that the thresholds for determining PD-L1 positivity have varied widely. In earlier studies of PD-L1 staining in DLBCL, a lower threshold, such as 5\% tumor positivity, was employed [5], similar to that used for the early studies of PD-L1 expression in melanoma [8]. In terms of predicting prognosis, it seems 
that higher expression levels, such as $30 \%$, as seen in the study by Kiyasu, et al, and our study, may be more relevant to identifying patients at risk of poorer clinical outcome. In our study, we found that lowering the cutoff threshold to 5,10 , or $20 \%$ resulted in non-statistically significant survival differences (data not shown). The study by Kwon, et al, further highlights the potentially tenuous relationship between PD-L1 expression and prognosis in DLBCL. A limitation to any proposed cutoff value for PD-L1 positivity is the inherently subjective nature of evaluating immunohistochemical staining. Computer aided detection and double-staining techniques may assist in the evaluation, but these approaches may not be practical for routine clinical use and carry their own limitations. Further complicating these analyses is the heterogeneous nature of the DLBCL, NOS category, and the inclusion of variants such as $\mathrm{EBV}+$ cases in the cohorts examined in the studies by Kiyasu, et al, and Kwon, et al. In our study, we have limited our cohort to EBV-negative DLBCL in an effort to exclude the influence of EBV positivity on prognosis. Nonetheless, several studies have found that PD-L1 expression in a wide range of different solid tumor types, including breast [26], bladder [27], stomach [28], and non-small cell lung carcinoma [29] correlates with poorer prognosis [30]. Clearly, better-powered studies involving larger patient populations receiving standardized chemotherapy regimens and long-term followup are needed to build upon these compelling findings.

The finding that a subset of DLBCL cases had PDL1 expression in non-malignant cells within the tumor microenvironment supports previous studies that observed a distinct proportion of classical Hodgkin lymphoma and DLBCL with this finding [5]. In our cohort, this finding did not translate into a significant prognostic factor, as was found by Kiyasu, et al [21]. The mechanisms responsible for the upregulation of $\mathrm{PD}-\mathrm{L} 1$ in the tumor microenvironment need further investigation, but are an intriguing source of tumor-induced immunomodulation that could potentially be targeted.

In the case of classical Hodgkin lymphoma, at least two independent genetic mechanisms, 9p24 amplification and EBV infection, are thought to lead to overexpression of PD-L1 in the malignant Reed-Sternberg cells [6, 7]. Similarly, the majority of EBV-positive aggressive B-cell lymphomas, including EBV-positive DLBCL, show upregulation of PD-L1 [5]. For DLBCL-NOS, a recent study identified a genetic basis of PD-L1 overexpression through translocations between the $P D-L 1$ and $I G H$ gene loci [31]. These cases may represent a distinct subtype of DLBCL that will require further characterization.

With the anticipation of additional clinical trials of immunotherapy directed at the PD-1 axis, treatment challenges will include determining the ideal time to initiate targeted immunotherapy; whether to combine immunotherapy with conventional chemotherapy, other immune checkpoint inhibitors, or hematopoietic stem cell transplant; and whether treatment should be continued following the first remission to prevent recurrence. Identification of DLBCL cases that express PD-L1 may form a rational basis for guiding therapy and can serve to select a subset of patients that could further benefit from targeted immunotherapy. Threshold levels of PD-L1 expression will need to be further examined to determine a biologically relevant level of expression that can predict tumor response to therapy and/or predict patient prognosis.

\section{MATERIALS AND METHODS}

\section{Case selection}

Cases of DLBCL, NOS, diagnosed between 2000 and 2014 were retrieved from the surgical pathology files and medical records of our institution. The study was approved by the University of Massachusetts Medical School Institutional Review Board. All DLBCLs were diagnosed and classified according to 2008 World Health Organization (WHO) criteria. Eighty-six DLBCL cases were included, composed of 46 female and 40 male patients, with a median age of 70 years (range 15-91 years). The primary sites involved by DLBCL in descending order of frequency included lymph node (32 cases), soft tissue (18 cases), spleen (5 cases), bone (4 cases), central nervous system (4 cases), skin (4 cases), salivary gland ( 3 cases), paranasal sinuses ( 3 cases), small bowel (3 cases), testis ( 3 cases), breast ( 2 cases), liver ( 2 cases), lung ( 2 cases), and bladder ( 1 case). All cases were negative for EBV-encoded RNA (EBER) by in situ hybridization study.

\section{Immunohistochemistry and evaluation}

Immunohistochemistry using a rabbit anti-PD-L1 monoclonal antibody (clone E1L3N, \#13684, Cell Signaling, Danvers, Massachusetts) was performed on $5 \mu \mathrm{m}$-thick, formalin-fixed paraffin embedded (FFPE) tissue sections and tissue microarray sections using a Dako Autostainer (Dako Corporation, Carpinteria, CA) with antigen retrieval methods $(0.01 \mathrm{M}$ citrate buffer at $\mathrm{pH}$ 6.0) as described previously [5]. The UltraView Universal DAB Detection kit (\#760-500, Ventana Medical Systems, Tuscon, AZ) was used according to the manufacturer instructions. Counterstaining was done as part of the automated staining protocol using hematoxylin (\#7602021, Ventana Medical Systems).

All IHC-stained sections were evaluated and scored by two hematopathologists independently. Discrepancies in scoring ( $<10 \%$ of cases) were resolved by consensus conference between the two pathologists. Threshold values of 30\% for BCL2, BCL6, CD10, and MUM1 staining were chosen reflecting routine clinical practice. MYC and Ki67 expression was recorded using a percentage scale 
of positive tumor ranging from $0 \%$ to $100 \%$. Staining intensity of PD-L1 was scored as follows: 0 (no staining), $1+$ (weak), 2+ (moderate), or 3+ (strong). Tumor cells exhibiting $2+$ or $3+$ membrane staining were recorded as a percentage of total tumor cellularity. PD-L1-positive nonmalignant cells as a percentage of total tumor cellularity was also recorded. Appropriate external positive (placenta) and negative (tonsil) controls were included with each staining run.

\section{Clinical data}

Patient demographics, clinical data, treatment and outcome information were obtained from the medical record. Clinical parameters included: presence of $\mathrm{B}$ symptoms, performance status, CBC, kidney and liver function tests, lactate dehydrogenase (LDH), bone marrow involvement, other extranodal sites involvement, bulky disease $(>10 \mathrm{~cm})$, Ann Arbor stage, International Prognostic Index (IPI), positron emission tomographycomputed tomography (PET/CT) scan at diagnosis and after treatment, treatment history, response to first treatment (complete remission, partial remission, stable disease and progressive disease), major complications to treatment (e.g., neutropenic fever, sepsis, chronic heart failure, renal failure, etc), the dates of disease progression, relapse or death and cause of death.

\section{Statistical analysis}

Comparisons of clinical data between groups were carried out using the Fisher exact test, Chi-Square test, and survival analysis in SAS v9.3 (SAS Software, Cary, NC, USA) and R 3.2.1. Progression-free survival (PFS) was measured from the date of original diagnosis to the date of last follow-up or of the first progression, relapse or death, toxicity events being excluded. Overall survival (OS) was measured from the date of original diagnosis to the date of last follow-up or death from any cause. PFS and OS were estimated using the Kaplan-Meier method. Differences of PFS or OS at different PD-L1 expression levels were assessed using the Log-Rank tests at the twosided significance level of 0.05 .

Age and sex-adjusted and multivariate analyses were conducted to evaluate the potential association between PD-L1 expression as well as other covariates with clinical outcomes. The stratified Cox proportional hazards model was used to determine hazard ratios and confidence intervals (CI) at the 95th confidence level. The variables included in multivariate analysis for OS were age, sex, expression of PD-L1, GCB/non-GCB type, stage and IPI. All of these variables had a P-value $<0.1$ in the age- and sex-adjusted analysis. Occasional missing values in the data set did not appear to present significant problems in the modeling strategy. The proportional hazard assumptions were satisfied in both age and sex- adjusted and multivariate analyses based on the log-log plots and Schoenfeld residuals method.

\section{CONFLICTS OF INTEREST}

The authors have disclosed that they have no significant relationships with, or financial interest in, any commercial companies pertaining to this article.

\section{FUNDING}

None.

\section{REFERENCES}

1. Armand P. Immune checkpoint blockade in hematologic malignancies. Blood. 2015;125:3393-400. doi:10.1182/ blood-2015-02-567453.

2. Francisco LM, Sage PT, Sharpe AH. The PD-1 pathway in tolerance and autoimmunity. Immunol Rev. 2010;236:21942. doi:10.1111/j.1600-065X.2010.00923.x.

3. Keir ME, Butte MJ, Freeman GJ, Sharpe AH. PD-1 and its ligands in tolerance and immunity. Annu Rev Immunol. 2008;26:677-704. doi:10.1146/annurev. immunol.26.021607.090331.

4. Freeman GJ, Long AJ, Iwai Y, Bourque K, Chernova T, Nishimura H, Fitz LJ, Malenkovich N, Okazaki T, Byrne MC, Horton HF, Fouser L, Carter L, et al. Engagement of the PD-1 immunoinhibitory receptor by a novel B7 family member leads to negative regulation of lymphocyte activation. J Exp Med. 2000;192:1027-34.

5. Chen BJ, Chapuy B, Ouyang J, Sun HH, Roemer MGM, Xu ML, Yu H, Fletcher CDM, Freeman GJ, Shipp MA, Rodig SJ. PD-L1 Expression Is Characteristic of a Subset of Aggressive B-cell Lymphomas and Virus-Associated Malignancies. Clin Cancer Res. 2013;19:3462-73. doi:10.1158/1078-0432.CCR-13-0855.

6. Green MR, Monti S, Rodig SJ, Juszczynski P, Currie T, O’Donnell E, Chapuy B, Takeyama K, Neuberg D, Golub TR, Kutok JL, Shipp MA. Integrative analysis reveals selective 9p24.1 amplification, increased PD-1 ligand expression, and further induction via JAK2 in nodular sclerosing Hodgkin lymphoma and primary mediastinal large B-cell lymphoma. Blood. 2010;116:3268-77. doi:10.1182/blood-2010-05-282780.

7. Green MR, Rodig S, Juszczynski P, Ouyang J, Sinha P, O’Donnell E, Neuberg D, Shipp MA. Constitutive AP-1 activity and EBV infection induce PD-L1 in Hodgkin lymphomas and posttransplant lymphoproliferative disorders: implications for targeted therapy. Clin Cancer Res. 2012;18:1611-8. doi:10.1158/1078-0432. CCR-11-1942.

8. Taube JM, Klein A, Brahmer JR, Xu H, Pan X, Kim JH, Chen L, Pardoll DM, Topalian SL, Anders RA. 
Association of PD-1, PD-1 ligands, and other features of the tumor immune microenvironment with response to anti-PD-1 therapy. Clin Cancer Res. 2014;20:5064-74. doi:10.1158/1078-0432.CCR-13-3271.

9. Ansell SM, Lesokhin AM, Borrello I, Halwani A, Scott EC, Gutierrez M, Schuster SJ, Millenson MM, Cattry D, Freeman GJ, Rodig SJ, Chapuy B, Ligon AH, et al. PD-1 blockade with nivolumab in relapsed or refractory Hodgkin's lymphoma. N Engl J Med. 2015;372:311-9. doi:10.1056/NEJMoa1411087.

10. Brahmer JR, Tykodi SS, Chow LQM, Hwu W-J, Topalian SL, Hwu P, Drake CG, Camacho LH, Kauh J, Odunsi K, Pitot HC, Hamid O, Bhatia S, et al. Safety and activity of anti-PD-L1 antibody in patients with advanced cancer. N Engl J Med. 2012;366:2455-65. doi:10.1056/ NEJMoa1200694.

11. Le DT, Uram JN, Wang H, Bartlett BR, Kemberling H, Eyring AD, Skora AD, Luber BS, Azad NS, Laheru D, Biedrzycki B, Donehower RC, Zaheer A, et al. PD-1 Blockade in Tumors with Mismatch-Repair Deficiency. N Engl J Med. 2015. doi:10.1056/NEJMoa1500596.

12. Topalian SL, Hodi FS, Brahmer JR, Gettinger SN, Smith DC, McDermott DF, Powderly JD, Carvajal RD, Sosman JA, Atkins MB, Leming PD, Spigel DR, Antonia SJ, et al. Safety, activity, and immune correlates of anti-PD-1 antibody in cancer. N Engl J Med. 2012;366:2443-54. doi:10.1056/NEJMoa1200690.

13. Armand P, Nagler A, Weller EA, Devine SM, Avigan DE, Chen Y-B, Kaminski MS, Holland HK, Winter JN, Mason JR, Fay JW, Rizzieri DA, Hosing CM, et al. Disabling immune tolerance by programmed death-1 blockade with pidilizumab after autologous hematopoietic stem-cell transplantation for diffuse large B-cell lymphoma: results of an international phase II trial. J Clin Oncol. 2013;31:4199206. doi:10.1200/JCO.2012.48.3685.

14. Berger R, Rotem-Yehudar R, Slama G, Landes S, Kneller A, Leiba M, Koren-Michowitz M, Shimoni A, Nagler A. Phase I safety and pharmacokinetic study of CT-011, a humanized antibody interacting with PD-1, in patients with advanced hematologic malignancies. Clin Cancer Res. 2008;14:3044 51. doi:10.1158/1078-0432.CCR-07-4079.

15. Westin JR, Chu F, Zhang M, Fayad LE, Kwak LW, Fowler N, Romaguera J, Hagemeister F, Fanale M, Samaniego F, Feng L, Baladandayuthapani V, Wang Z, et al. Safety and activity of PD1 blockade by pidilizumab in combination with rituximab in patients with relapsed follicular lymphoma: a single group, open-label, phase 2 trial. Lancet Oncol. 2014;15:69-77. doi:10.1016/ S1470-204570551-5.

16. Hans CP, Weisenburger DD, Greiner TC, Gascoyne RD, Delabie J, Ott G, Müller-Hermelink HK, Campo E, Braziel RM, Jaffe ES, Pan Z, Farinha P, Smith LM, et al. Confirmation of the molecular classification of diffuse large B-cell lymphoma by immunohistochemistry using a tissue microarray. Blood. 2004;103:275-82. doi:10.1182/ blood-2003-05-1545.

17. Xu-Monette ZY, Dabaja BS, Wang X, Tu M, Manyam GC, Tzankov A, Xia Y, Zhang L, Sun R, Visco C, Dybkaer K, Yin L, Chiu A, et al. Clinical features, tumor biology, and prognosis associated with MYC rearrangement and Myc overexpression in diffuse large B-cell lymphoma patients treated with rituximab-CHOP. Mod Pathol. 2015;28:155573. doi:10.1038/modpathol.2015.118.

18. Petrich AM, Gandhi M, Jovanovic B, Castillo JJ, Rajguru S, Yang DT, Shah KA, Whyman JD, Lansigan F, HernandezIlizaliturri FJ, Lee LX, Barta SK, Melinamani S, et al. Impact of induction regimen and stem cell transplantation on outcomes in double-hit lymphoma: a multicenter retrospective analysis. Blood. 2014;124:2354-61. doi:10.1182/blood-2014-05-578963.

19. Johnson NA, Slack GW, Savage KJ, Connors JM, BenNeriah S, Rogic S, Scott DW, Tan KL, Steidl C, Sehn LH, Chan WC, Iqbal J, Meyer PN, et al. Concurrent expression of MYC and BCL2 in diffuse large B-cell lymphoma treated with rituximab plus cyclophosphamide, doxorubicin, vincristine, and prednisone. J Clin Oncol. 2012;30:3452-9. doi:10.1200/JCO.2011.41.0985.

20. Snuderl M, Kolman OK, Chen Y-B, Hsu JJ, Ackerman AM, Dal Cin P, Ferry JA, Harris NL, Hasserjian RP, Zukerberg LR, Abramson JS, Hochberg EP, Lee H, et al. B-cell lymphomas with concurrent IGH-BCL2 and MYC rearrangements are aggressive neoplasms with clinical and pathologic features distinct from Burkitt lymphoma and diffuse large B-cell lymphoma. Am J Surg Pathol. 2010;34:327-40. doi:10.1097/PAS.0b013e3181cd3aeb.

21. Kiyasu J, Miyoshi H, Hirata A, Arakawa F, Ichikawa A, Niino D, Sugita Y, Yufu Y, Choi I, Abe Y, Uike N, Nagafuji $\mathrm{K}$, Okamura $\mathrm{T}$, et al. Expression of programmed cell death ligand 1 is associated with poor overall survival in patients with diffuse large B-cell lymphoma. Blood. 2015. doi:10.1182/blood-2015-02-629600.

22. Herbst RS, Soria J-C, Kowanetz M, Fine GD, Hamid O, Gordon MS, Sosman JA, McDermott DF, Powderly JD, Gettinger SN, Kohrt HEK, Horn L, Lawrence DP, et al. Predictive correlates of response to the anti-PD-L1 antibody MPDL3280A in cancer patients. Nature. 2014;515:563-7. doi:10.1038/nature14011.

23. Andorsky DJ, Yamada RE, Said J, Pinkus GS, Betting DJ, Timmerman JM. Programmed death ligand 1 is expressed by non-hodgkin lymphomas and inhibits the activity of tumor-associated T cells. Clin Cancer Res. 2011;17:423244. doi:10.1158/1078-0432.CCR-10-2660.

24. Xerri L, Chetaille B, Serriari N, Seriari N, Attias C, Guillaume Y, Arnoulet C, Olive D. Programmed death 1 is a marker of angioimmunoblastic T-cell lymphoma and B-cell small lymphocytic lymphoma/chronic lymphocytic leukemia. Hum Pathol. 2008;39:1050-8. doi:10.1016/j. humpath.2007.11.012. 
25. Kwon D, Kim S, Kim P-J, Go H, Nam SJ, Paik JH, Kim YA, Kim TM, Heo DS, Kim CW, Jeon YK. Clinicopathological analysis of programmed cell death-1 and programmed cell death-ligand 1 expression in the tumor microenvironments of diffuse large B-cell lymphomas. Histopathology. 2015. doi:10.1111/his.12882.

26. Qin T, Zeng Y-D, Qin G, Xu F, Lu J-B, Fang W-F, Xue C, Zhan J-H, Zhang X-K, Zheng Q-F, Peng R-J, Yuan Z-Y, Zhang L, et al. High PD-L1 expression was associated with poor prognosis in 870 Chinese patients with breast cancer. Oncotarget. 2015;6:33972-81. doi:10.18632/ oncotarget.5583.

27. Huang Y, Zhang S-D, McCrudden C, Chan K-W, Lin $\mathrm{Y}$, Kwok H-F. The prognostic significance of PD-L1 in bladder cancer. Oncol Rep. 2015;33:3075-84. doi:10.3892/ or.2015.3933.

28. Eto S, Yoshikawa K, Nishi M, Higashijima J, Tokunaga T, Nakao T, Kashihara H, Takasu C, Iwata T, Shimada
M. Programmed cell death protein 1 expression is an independent prognostic factor in gastric cancer after curative resection. Gastric Cancer. 2015. doi:10.1007/ s10120-015-0519-7.

29. Pan Z-K, Ye F, Wu X, An H-X, Wu J-X. Clinicopathological and prognostic significance of programmed cell death ligand1 (PD-L1) expression in patients with non-small cell lung cancer: a meta-analysis. J Thorac Dis. 2015;7:462-70. doi:10.3978/j.issn.2072-1439.2015.02.13.

30. Jin Y, Zhao J, Shi X, Yu X. Prognostic value of programed death ligand 1 in patients with solid tumors: A meta-analysis. J Cancer Res Ther. 2015;11:C38-43. doi:10.4103/0973-1482.163837.

31. Georgiou K, Chen L, Berglund M, Ren W, de Miranda NFCC, Lisboa S, Fangazio M, Zhu S, Hou Y, Wu K, Fang W, Wang X, Meng B, et al. Genetic basis of PD-L1 overexpression in diffuse large B-cell lymphomas. Blood. Epub ahead of print. doi:10.1182/blood-2015-12-686550. 